\title{
Tumor microenvironment as a potential source of clinical biomarkers in non-small cell lung cancer: can we use enemy territory at our advantage?
}

\author{
Carlo Genova $^{1,2}$, Erika Rijavec $^{1}$, Francesco Grossi ${ }^{1}$ \\ ${ }^{1}$ Lung Cancer Unit, Ospedale Policlinico San Martino, Genoa, Italy; ${ }^{2}$ Department of Internal Medicine and Medical Specialties (DIMI), University \\ of Genoa, Genoa, Italy \\ Correspondence to: Dr. Francesco Grossi, MD. Lung Cancer Unit, Ospedale Policlinico San Martino, Genoa, Italy. Email: francesco.grossi@hsanmartino.it. \\ Provenance: This is an Invited Editorial commissioned by Section Editor Dr. Long Jiang (Department of Thoracic Oncology, Second Affiliated \\ Hospital, Institute of Respiratory Diseases, Zhejiang University School of Medicine, Hangzhou, China). \\ Common on: Gocheva V, Naba A, Bhutkar A, et al. Quantitative proteomics identify Tenascin-C as a promoter of lung cancer progression and \\ contributor to a signature prognostic of patient survival. Proc Natl Acad Sci U S A 2017;114:E5625-34.
}

Submitted Oct 05, 2017. Accepted for publication Oct 13, 2017.

doi: $10.21037 /$ jtd.2017.10.66

View this article at: http://dx.doi.org/10.21037/jtd.2017.10.66

The relevance of tumor microenvironment (TME) in tumorigenesis is widely known, and many efforts have been made in order to fully understand its peculiarities (1). It is acknowledged that tumor cells closely interact with TME, which is constituted by blood vessels, immune cells, fibroblasts, signaling molecules, and the extracellular matrix (ECM). On one hand, tumor cells modify the microenvironment by releasing extracellular signals, thus promoting immune tolerance and neoangiogenesis, resulting in an ecosystem that is suitable for their survival; on the other hand, the now-adapted TME promotes proliferation and survival of neoplastic cells $(2,3)$. This complex interaction between tumor cells and the surrounding environment has been the subject of several preclinical and clinical studies and has provided potential biomarkers for clinical practice as well as targets for antineoplastic therapies; this is of particular relevance, among other malignancies, in non-small cell lung cancer (NSCLC), as improvements in its management represent an urgent medical need. As an example, tumor cells induce neoangiogenesis by paracrine signaling directed on stromal fibroblasts and endothelial cells; therefore, vascular-disrupting agents, such as bevacizumab, represent a generally successful therapeutic approach $(4,5)$. As immune checkpoint blockade has become a therapeutic standard in several solid tumors, including NSCLC $(6,7)$, the interest in the characterization of tumor-infiltrating immune cells has significantly grown. Indeed, the number of specific tumor-infiltrating immune cell subpopulations, such as FOXP3 ${ }^{+}$Treg cells, has been reported as a potential prognostic factor in NSCLC (8); furthermore, it has been suggested that tumor samples of non-smoking patients with lung adenocarcinoma are characterized by an immunosuppressive microenvironment, which might promote tumor progression (9). In addition to immune and stromal cells, TME is characterized by its non-cellular component, globally known as the ECM; this entity contains a complex set of molecules, usually secreted by stromal cells, which are pivotal in the maintenance of tissue architecture in normal conditions. Notably, TME can also affect tumor cell proliferation, potentially favoring cell invasion and development of metastases, while the presence of neoplastic cells is known to modify the composition of TME in return (10). In spite of the critical role of ECM in cancer, its modifications in presence of tumor cells are not completely known and represent an emerging subject of interest for basic and clinical research (11). Due to its tight relationship with tumor cells, ECM has been considered a promising source of potential cancer biomarkers for a long time (12). In normal conditions, ECM is highly organized and its characteristics depend on the precise proportion of its components, which ultimately define its physical and biochemical properties. Features such as porosity, elasticity, 
solubility, and orientation are pivotal for determining how ECM supports normal tissue growth and maintenance of architecture; furthermore, ECM can limit the accessibility of receptors on cell surface to soluble ligands such as growth factors due to its bioelectrical charge, or even generate active signaling ligands starting from its own components. Notably, ECM composition may vary dramatically among different organs and tissues. While ECM is physiologically characterized by constant remodeling and by strong bilateral interactions with normal cells, the presence of neoplastic cells might result in aberrant disposition of its components and ultimately disruption of its normal characteristics and functions $(13,14)$. Some of the proteins that can be found in ECM are significantly associated with tumorigenesis and include, among many others, collagen (its most frequently found component), elastin, fibronectin, periostin, and tenascin-C; these proteins are crucial for the development of stroma in normal conditions and have a relevant role in epithelial-to-mesenchymal transition, proliferation, and migration of cancer cells.

Notably, some of these proteins appear to be particularly worth of mention as potential biomarkers of clinical interest in oncology. For example, a fibronectin isoform containing a specific extra domain (ED-B) tends to accumulate around newly formed blood vessels where physiologic or cancerrelated neoangiogenesis takes place, but not around mature blood vessels; interestingly, it has been demonstrated that the quantification of this specific isoform with a human recombinant antibody in astrocytoma samples was able to accurately discriminate between high-grade and lowgrade astrocytoma (15). Another ECM-affiliated protein, periostin, is known to be a cell adhesion molecule and to play a remarkable role in epithelial-to-mesenchymal transition; this protein has been associated with outcomes in different malignancies, including prostate and breast cancer (16). Finally, tenascin- $\mathrm{C}$ is an adhesion modulatory molecule found in ECM, which is highly expressed during normal tissue repair and in many solid tumors; this protein has been shown to be significantly associated with prognosis in several solid tumors, including astrocytic tumors and breast cancer (14,17-19). Notably, neoplastic cells are not only able to increase the expression of tenascin- $\mathrm{C}$, but also influence its qualitative composition; indeed, while extracellular $\mathrm{pH}$ appears to influence the alternative splicing of RNA encoding for tenascin-C produced by normal fibroblasts, resulting in different proportion of its isoforms, malignant cells do not seem to be influenced by external $\mathrm{pH}$ and they mostly produce the large isoform of this protein (20).
Most recently, a team of researchers from Cambridge focused on the study of ECM changes in lung cancer; since cell culture models were not suitable for studying the TME, they employed a specifically engineered mouse model designed to reproduce the complexity of lung adenocarcinoma within its microenvironment. In order to evaluate the numerous extracellular components of the TME, the researchers employed an approach based on quantitative proteomics, which allows a comprehensive characterization of the complex of ECM and its associated proteins, collectively defined as "matrisome". According to this approach, after removing intracellular components, the ECM-enriched samples underwent mass spectrometry and the resulting spectra are then interpreted with specific bioinformatics software. The researchers used this technology to analyze primary lung cancer in their mouse model and compare it with metastatic mediastinal lymph nodes, lung fibrosis, and healthy lung tissue; more specifically, mediastinal lymph node involvement was considered as a sign of cancer progression at a more advanced stage, while lung fibrosis (induced by bleomycin in the mouse model) was chosen as it is a disease characterized by excessive deposition of ECM, resulting in the disruption of the normal extracellular structures of the lung. The mass spectrometry analysis revealed a large number of peptides, which were associated with $113 \mathrm{ECM}$-affiliated proteins. Subsequently, an independent component analysis detected three different ECM signatures, which were specifically associated with fibrosis, primary tumors and metastases; the signatures were then analyzed by using volcano plots in order to highlight those ECM-affiliated proteins that were present in different quantity across the different samples. Tenascin-C and fibronectin were significantly increased in fibrotic and neoplastic samples compared to healthy lung, while three members of the S100 family (S100A6, S100A10, S100A11) were enriched in tumor samples, but not in fibrotic lung, compared to healthy samples. Notably, only few proteins were significantly different between neoplastic and fibrotic samples and between primary tumors and lymph node metastases. Subsequently, the researchers performed immunohistochemistry (IHC) assessment of some identified ECM-affiliated proteins in order to validate the mass spectrometry findings, thus confirming increased expression of S100A6, S100A10, and S100A11 in tumor samples (including primary tumor and lymph nodal metastases), as well as significantly increased expression of fibronectin and tenascin-C in fibrotic and neoplastic lungs as compared to normal lungs. Then, the researchers focused on tenascin- $\mathrm{C}$ 
on the hypothesis of a functional role of this protein in the development of fibrosis and neoplastic progression. Initially, they determined the gene expression of tenascin- $C$ in cell lines collected from non-metastatic lung tumors, metastatic lung tumors, and metastases, observing that the non-metastatic tumors had significantly lower expression of the tenascin- $C$ gene compared to more advanced tumors; subsequently, they identified a correlation between the progressive loss of activity of the oncosuppressor Nkx2-1 and increased tenascin- $C$ expression in advanced tumors. Additionally, when tenascin- $C$ RNA-overexpressing cell lines were inoculated in mice, it was observed that its overexpression was associated with increased risk of developing metastases, rather than influencing primary tumor growth. In a subsequent step, the exploration of the Cancer Genome Atlas (TCGA) lung adenocarcinoma cohort revealed increased RNA expression of tenascin- $C$, S100A6, and S100A11 in lung cancer compared to normal lungs across the dataset and compared to matched normal tissue from patients affected by lung cancer; furthermore, high expression of tenascin-C, S100A10 and S100A11 RNAs were associated with poor prognosis in lung cancer, thus allowing to generate a three-gene prognostic signature that appears to be specific for lung cancer (21).

The reported work is particularly remarkable due to its approach, which took into account the vast complexity of the ECM and the resulting difficulties in achieving robust and meaningful findings. Indeed, the authors started by performing a wide analysis of the mass spectra observed in neoplastic and in healthy samples, and subsequently selected the potential biomarkers for further analyses. The mass spectrometry approach is able to screen a relevant number of peptides even in a limited number of samples, and hence potentially provide a huge amount of raw information; notably, mass spectrometry has already been employed in clinical studies designed to detect potential prognostic or predictive signatures in patients affected by advanced NSCLC, and this approach led to the identification of two distinct signatures able to discriminate between patients with good and poor outcomes while receiving chemotherapy or targeted agents; notably, such signatures were detected in plasma, which is often referred as another potential source of biomarkers, considered promising due to its easy accessibility $(22,23)$. Mass spectrometry falls among those approaches able to simultaneously study multiple targets within a relatively limited amount of samples, which also include, above others, next generation sequencing and tissue microarrays (24). While these high-throughput techniques are generating an impressive amount of data, some potential pitfalls of this approach should be addressed; this is especially true when rich sources of potential biomarkers, such as the ECM, are screened in this way, and the authors of the reported study put a significant effort in this part of their research. In first place, the non-negligible possibility of identifying by chance statistically significant differences needs to be ruled out; hence, selecting the most relevant potential biomarkers and subsequently validating them represent critical steps in the generation of robust results. The first task is required to reduce the excessive amount of generated raw data (e.g., genes identified with a microarray; mass spectrometry ECM-peptide profiles) and limit the following experiments to the actually relevant molecules. Additionally, as the magnitude of the observed results might still be overestimated (e.g., due to limited sample size), data validation with different techniques or datasets, such as additional samples or patients' cohorts, is pivotal for increasing the robustness of the findings. Another potential issue lies in the demonstration of the mechanisms of causeeffects underlying any observed predictive or prognostic biomarker; while a passenger mutation or increased protein expression identified as a biomarker may just be an epiphenomenon correlated with response to treatment or survival, a causal relationship has to be determined or ruled out in order to increase basic and translational knowledge and to define whether the candidate biomarker might be a potential therapeutic target. Finally, the translational relevance of any preclinical result needs solid confirmation in the clinical setting, where other parameters with acknowledged predictive or prognostic value have to be taken into account.

In addition to the issues determined by the sheer amount of ECM components and by the requirements for generating robust data, we should not neglect that ECM is intrinsically dynamic, constantly being remodeled on the basis of paracrine signaling or even environmental alterations [for example, the alternative splicing of tenascin- $C$ pre-mRNA can lead to a different composition of protein isoforms, some of which are closely associated with malignant transformation (25)]; furthermore, the possible alterations of a specific ECM-affiliated protein with a potential predictive/prognostic role might depend not only on its own quantity, but also on its possible interactions with other proteins of matrisome, which themselves might be aberrantly expressed (13).

In summary, the complexity of the ECM in physiologic conditions and its interactions with cancer cells translate 
into the requirement of sophisticated and comprehensive studies in order to assess the translational role of its components in carcinogenesis and as predictive or prognostic factors. While the impressive amount of ECMaffiliated proteins ecosystem poses a relevant challenge to researchers, an increasing knowledge of the physiologic ECM constituents and of how neoplastic cells modify ECM might result in the definition of biomarkers of translational relevance and eventually novel targets for antineoplastic treatments.

\section{Acknowledgements}

None.

\section{Footnote}

Conflicts of Interest: The authors have no conflicts of interest to declare.

\section{References}

1. Stadler M, Walter S, Walzl A, et al. Increased complexity in carcinomas: Analyzing and modeling the interaction of human cancer cells with their microenvironment. Semin Cancer Biol 2015;35:107-24.

2. Spill F, Reynolds DS, Kamm RD, et al. Impact of the physical microenvironment on tumor progression and metastasis. Curr Opin Biotechnol 2016;40:41-8.

3. Kerbel RS. A Decade of Experience in Developing Preclinical Models of Advanced- or Early-Stage Spontaneous Metastasis to Study Antiangiogenic Drugs, Metronomic Chemotherapy, and the Tumor Microenvironment. Cancer J 2015;21:274-83.

4. Watnick RS. The role of the tumor microenvironment in regulating angiogenesis. Cold Spring Harb Perspect Med 2012;2:a006676.

5. Aita M, Fasola G, Defferrari C, et al. Targeting the VEGF pathway: antiangiogenic strategies in the treatment of non-small cell lung cancer. Crit Rev Oncol Hematol 2008;68:183-96.

6. Rijavec E, Genova C, Alama A, et al. Role of immunotherapy in the treatment of advanced non-smallcell lung cancer. Future Oncol 2014;10:79-90.

7. Dal Bello MG, Alama A, Coco S, et al. Understanding the checkpoint blockade in lung cancer immunotherapy. Drug Discov Today 2017;22:1266-73.

8. Shimizu K, Okita R, Nakata M. Clinical significance of the tumor microenvironment in non-small cell lung cancer. Ann Transl Med 2013;1:20.

9. Kinoshita T, Kudo-Saito C, Muramatsu R, et al. Determination of poor prognostic immune features of tumour microenvironment in non-smoking patients with lung adenocarcinoma. Eur J Cancer 2017;86:15-27.

10. Pickup MW, Mouw JK, Weaver VM. The extracellular matrix modulates the hallmarks of cancer. EMBO Rep 2014;15:1243-53.

11. Denys H, Braems G, Lambein K, et al. The extracellular matrix regulates cancer progression and therapy response: implications for prognosis and treatment. Curr Pharm Des 2009;15:1373-84.

12. Sund M, Kalluri R. Tumor stroma derived biomarkers in cancer. Cancer Metastasis Rev 2009;28:177-83.

13. Lu P, Weaver VM, Werb Z. The extracellular matrix: a dynamic niche in cancer progression. J Cell Biol 2012;196:395-406.

14. Frantz C, Stewart KM, Weaver VM. The extracellular matrix at a glance. J Cell Sci 2010;123:4195-200.

15. Castellani P, Borsi L, Carnemolla B, et al. Differentiation between high- and low-grade astrocytoma using a human recombinant antibody to the extra domain-B of fibronectin. Am J Pathol 2002;161:1695-700.

16. Nuzzo PV, Rubagotti A, Zinoli L, et al. The prognostic value of stromal and epithelial periostin expression in human breast cancer: correlation with clinical pathological features and mortality outcome. BMC Cancer 2016;16:95.

17. Bonnans C, Chou J, Werb Z. Remodelling the extracellular matrix in development and disease. Nat Rev Mol Cell Biol 2014;15:786-801.

18. Viale G, Dorcaratto A, Castellani P, et al. Tenascin-C in astrocytic tumors. Surg Neurol 2002;57:286; author reply 7.

19. Yang $Z$, Ni W, Cui C, et al. Tenascin $C$ is a prognostic determinant and potential cancer-associated fibroblasts marker for breast ductal carcinoma. Exp Mol Pathol 2017;102:262-7.

20. Borsi L, Allemanni G, Gaggero B, et al. Extracellular pH controls pre-mRNA alternative splicing of tenascin-C in normal, but not in malignantly transformed, cells. Int $\mathrm{J}$ Cancer 1996;66:632-5.

21. Gocheva V, Naba A, Bhutkar A, et al. Quantitative proteomics identify Tenascin-C as a promoter of lung cancer progression and contributor to a signature prognostic of patient survival. Proc Natl Acad Sci U S A 2017;114:E5625-34.

22. Grossi F, Rijavec E, Genova C, et al. Serum proteomic test in advanced non-squamous non-small cell lung cancer 
treated in first line with standard chemotherapy. Br J

Cancer 2017;116:36-43.

23. Gregorc V, Novello S, Lazzari C, et al. Predictive value of a proteomic signature in patients with non-small-cell lung cancer treated with second-line erlotinib or chemotherapy (PROSE): a biomarker-stratified, randomised phase 3 trial. Lancet Oncol 2014;15:713-21.

Cite this article as: Genova C, Rijavec E, Grossi F. Tumor microenvironment as a potential source of clinical biomarkers in non-small cell lung cancer: can we use enemy territory at our advantage? J Thorac Dis 2017;9(11):4300-4304. doi: 10.21037/ jtd.2017.10.66
24. Vanni I, Coco S, Truini A, et al. Next-Generation Sequencing Workflow for NSCLC Critical Samples Using a Targeted Sequencing Approach by Ion Torrent PGM Platform. Int J Mol Sci 2015;16:28765-82.

25. Silacci M, Brack SS, Spath N, et al. Human monoclonal antibodies to domain $\mathrm{C}$ of tenascin-C selectively target solid tumors in vivo. Protein Eng Des Sel 2006;19:471-8. 